\title{
An Empiricist Criterion of Meaning
}

\author{
Yann Benétreau-Dupin \\ Center for Philosophy and History of Science \\ Boston University \\ yannbd@bu.edu
}

\section{Empirical Meaning and Scientific Realism.}

When we use scientific propositions, in order to describe phenomena and organize our knowledge about the physical world, we use terms that do not refer to observable entities. So as to explain chemical reactions, we would refer to atoms or electrons, that we cannot see directly. And even such a familiar concept as the one of mass is not observable, in the sense that it cannot be in all cases, in all experimental contexts, defined only by observable concepts or observations. Consequently, we can worry about the role of such theoretical terms, and wonder what their empirical meaning is, or at least whether it is possible to find a way to express such an empirical meaning. Empiricists may ask that theoretical terms should be used only if they are empirically meaningful. The question I wish to deal with is one of knowing what theoretical terms refer to, and to what extent we are committed to the existence of theoretical entities by adopting a theory. I wish to show that it is possible to characterize the empirical meaning of theoretical terms and address the worry of their ontological commitment. 
There is an ongoing debate over whether or not the entities posited by successful scientific theories are real. There are two competing arguments: one the one hand, according to the no-miracles argument, "[ $\mathrm{t}]$ he positive argument for realism is that it is the only philosophy that doesn't make the success of science a miracle." (Putnam, 1975, p. 73) On the other hand, according to the pessimistic meta-induction, history of science has repeatedly shown us that belief in the reality of theoretical entities is more likely to turn into revocation: all theoretical constructs had to be greatly modified or abandoned, and there is no reason to think that our present knowledge will not need major amendments (see e.g., Laudan,1981, or McMullin, 1984).

As a way to overcome this impasse, and in order to save as much of scientific realism as possible, taking into account important theoretical changes in science, John Worrall (1989) introduced a philosophical position called structural realism. According to Worrall, the pessimistic induction argument prevents us from thinking that our best theories cuts nature at its joints, and then from accepting scientific realism; and the success of science prevents us from being antirealists. In any theory change, Worrall claims, even though the reference to some theoretical entities is abandoned, there is "continuity or accumulation in the shift, but the continuity is one of form or structure, not of content."' (1989, p. 117, original emphasis) According to this position, what we should be realist about is "relationships between phenomena expressed in the mathematical equations of (the) theor(ies), the theoretical terms of which should be understood as

1 Here, Worrall takes the example of the transition in nineteenth-century optics from Fresnel's elastic solid ether theory to Maxwell's theory of the electromagnetic field, in which, he argues, the identification of the structure of light remained unaltered. 
genuine primitives." (Worrall, 1989, p. 122) Any past theories, even if they were later refuted, as for instance was the theory of ether, identified relationships between phenomena that remained valid throughout theory changes.

This position, which was thought by Worrall to be "the best of both worlds" (1989), has since been the object of an important literature (see e.g., Ladyman, 1998, Psillos, 1999, the latter arguing for scientific realism). A version of structural realism has been put forth by Otávio Bueno (1999, 2010):

The proposal shares with structural realism the emphasis on structure. But it completely abandons realism. Structures are employed not to capture the "structure of the world"; they only help us represent what is observable via the formulation of empirically adequate (or quasi-true) theories. Thus, the account sides with an empiricist interpretation, and can be called structural empiricism (see Bueno [1999]). (Bueno, 2010, p. 14)

In the debate about structural realism, it suggests to view the success of science as its ability to "capture the structure of the world" (ibid.), with structures that can survive theory change, and yet not make ontological assumptions as to the reality of theoretical entities that are necessary to scientific inquiry. Thereby, it hopes to take into account both the no-miracles argument, by identifying permanent structural relationships between observables, and the pessimistic induction argument, by assessing theories only regarding their empirical adequacy, and avoiding the question of the ontological commitment to unobservable entities.

In line with the intent of this proposal, I wish here to defend an empiricist interpretation of structuralism, while avoiding commitment to realism with regards to unobservable, theoretical entities. I wish to find a way to express how theories impose 
structural relationships between observables. In other words, I wish to present a way to express the semantical import of theoretical terms, in terms of observables only. I will do so by reconsidering Frank Ramsey's method of replacing theoretical terms by relationships between observational terms. This method has be seen as "the purest structuralism possible" (Laudan, 1998, p. 412) but faced important criticisms.

We will see that it implies a second-order quantification that, instead of truly eliminating theoretical entities, implies a possible greater ontological commitment to theoretical entities. Ramsey's method has also met another important objection: M. H. A. Newman (1928) pointed out their triviality, the fact that a Ramsey elimination merely imposes a constraint on the cardinality of its domain, which is not a constraint at all.

Both these criticisms can be overcome; solutions had been formulated to show that Ramsey sentences could exhibit the role of theories in a way that could receive a first-order equivalent, thus expressing a structure - a functional dependence - between observables.

I wish to show that theoretical concepts can play a genuine role, receive an empirical meaning, and yet not imply that we should be realist as to their existence. This can show that Ramsey sentences constitute a relevant tool to assess the empirical meaning of theoretical sentences, and constitute an interesting tool for empiricists that can resist the criticisms that had been previously raised against it.

To do so, I wish to point out that it is enough for theoretical terms to put a constraint on observables for them to be empirically meaningful. This is precisely what 
Ramsey's method does, and can be shown by invoking results from William Craig's replacement program (1960).

Then, in order to show that Ramsey's method can constitute a way to express the role of theoretical entities and, at the same time, allows one to remain agnostic as to their existence, I will have to counter Stathis Psillos' criticism against the inability for empiricists to reduce the truth-conditions of theoretical sentences to truth-conditions on observables only. Or, as he puts it:

Semantic realists treat [theoretical]-discourse as having truth-conditions which are irreducible in that the referred-to entities themselves are irreducible and the truth-conditions of an assertion as a whole are not reducible to evidence - or verification conditions. (Psillos, 1999, p. 14-15)

I will then have to show that the effect theoretical terms impose on observational propositions can be expressed in a first-order logic, and receive first-order truthconditions.

Works by Jaakko Hintikka (1996, 1998 and 2009) have shown how the use of adequate rule for a first-order logic allow one to find a first-order equivalent to secondorder Ramsey sentences. I can here use them to counter Psillos' criticisms against the empiricists' attempt to express the role of theoretical terms and, at the same time "withhold belief in anything that goes beyond the actual, observable phenomena" (Van Fraassen, 1980, p. 202) It is the possibility to consider Ramsey-elimination as an empiricist tool that I wish to show here. 


\section{Expressing the Role of Theories in Terms of Observable Consequences: Ramsey's Original Proposal.}

In his Physics: The Elements (1920), N. R. Campbell had proposed to distinguish between theoretical language and observational language. The latter contains empirical data and physical laws that are generalizations of empirical regularities. For instance, Kepler's third law (that asserts that, for two given celestial bodies, there is a constant ratio between their period of revolution squared and their relative distance cubed) is an empirical law. Such laws "mean nothing but what they assert" (Campbell, 1920, p. 132), i.e., they only express a regularity between empirical magnitudes. Contrary to laws, theoretical terms are meaningful within a theory that makes assumptions over entities that do not belong to the realm of observation. For instance, the term 'mass' is part of a whole set of theoretical assumptions. If we were to try to define it strictly as the expression of a relationship between observable properties, we would face an important obstacle. Ernst Mach declared about Newton's definition of mass:

With regard to the conception of 'mass', it is to be observed that the formulation of Newton, which defines mass to be the quantity of matter of a body as measured by the product of its volume and density, is unfortunate. As we can only define density as the mass of a unit volume, the circle is manifest. $^{2}$ (Mach, 1883, chap. 2, sect. 3, paragraph 7)

This impossibility to define explicitly 'mass' in terms of a relationship between observable properties makes it, in Campbell's proposition, part of the theoretical language. In this sense, it belongs to a theoretical language, and is related to theoretical

2 Quoted in Jammer (2000). 
laws that do not only exclusively express relationships between observables. Theoretical laws are "general hypotheses about how the world works" (Sahlin, 1990, p. 130). Theoretical terms, such as 'mass', that can be used in a law, are meaningful within a theory that makes assumptions over entities that do not belong to the realm of observation - it constitutes the basis of the judgments whose regularities are expressed by laws. ${ }^{3}$ Their meaningfulness lies in their possible connection with empirical laws. A 'dictionary' can be considered as giving a correspondence between a theoretical term, the 'entry of the dictionary' to be explained and an observational term, proposition or set of propositions, that can be verifiable observable magnitudes. ${ }^{4}$

Keeping further Campbell's idea of two distinct languages linked by a dictionary, ${ }^{5}$ Ramsey, in "Theories" (1929), showed that, even though it would be possible in principle to establish a definite correspondence between these two languages by means of explicit definitions, it is neither useful nor desirable. For this purpose, Ramsey does not consider how to express the theoretical in terms of the observable, but rather to go the other way

3 For instance, Newton's mechanical theory introduced a set of axioms to explain regularities among phenomena, through abstract entities such as force or mass, that cannot be defined explicitly in terms of observable entities or properties (such as "red", "iron", "table", etc.) but that can however be identified through their effects and whose magnitude can be measured.

4 Campbell puts it as forth: "[a] theory is a connected set of propositions which are divided into two groups. One group consists of statements about some collection of ideas which are characteristic of the theory; the other group consists of statements of the relation between these ideas and and some other ideas of a different nature. The first group will be termed collectively the "hypothesis" of the theory; the second group the "dictionary". The hypothesis is so called (...) because the propositions composing it are incapable of proof or disproof by themselves; they must be significant, but, taken apart from the dictionary, they appear arbitrary assumptions. (...) The ideas which are related by means of the dictionary to the ideas of the hypothesis are, on the other hand, such that something is known about them apart from the theory. It must be possible to determine, apart from the theory, whether certain propositions involving these ideas are true or false." (Campbell, 1920, p. 122)

5 Ramsey does refer explicitly to Campbell's work, but his article "Theories" is obviously related to Campbell's considerations in the $\mathrm{XX}^{\text {th }}$ chapter of his Physics: The Elements. 
around, with an inverted dictionary. In this sense, "a theory [can be described] as a language for discussing the facts the theory is said to explain." (Ramsey, 1929) ${ }^{6}$

If it is not interesting to look for a strict correspondence between observational and theoretical terms, for Ramsey it is because by doing so we would lose the dynamic character of theories: if we reach an explicit definition of a theoretical term, and if this definition needs to be amended (e.g., after new data or laws have been discovered), we can extend the definition, by adding a property to the definition of a term. But this would not be possible if we used only explicit definitions, or we could not amend without changing it entirely.

It must be said clearly that the possibility that the theoretical could be defined by the observable has to be ruled out. Let us take an example to see why this cannot hold. We may, as we did earlier, consider the term of 'mass' as a theoretical one. Now, consider what would happen if we tried to specify experimental processes by which we could measure a value of mass. If for instance we want to find out the mass of an electron, we could do it by measuring the deflection of this electron's trajectory by a given electromagnetic field. If now I want to find out the mass of a planet, I can do so by observing how its satellites orbit around it, and measure their period of revolution and how far away they are from their planet. ${ }^{7}$ In both cases, we would have a definite relationship between mass, and observable, measurable data. However, these two

6 This way of investigation seems to correspond more naturally to Campbell's view according to which a theory explains a law (which deals with empirical generalization), as well as we would reformulate in a new language a statement that we would want to explain, originally expressed in an less familiar language.

7 Thanks to Kepler's third law. 
relationships, the formula that relate 'mass' to the other magnitudes, are not the same. The impossibility to find a single correspondence between the concept of mass and observable data leads us to abandon the idea of its definability in terms of observables only. ${ }^{8}$

The empirical meaningfulness of a theoretical concept does not lie in its ability to be defined by observable measuring processes. Such processes are always limited to given experimental contexts. However, it is not necessary for a theoretical concept to be definable in terms of observables to be meaningful. Even though a value of mass is obtained in different ways in different contexts, the concept of mass can be said to be empirically meaningful because, broadly speaking, it says something about observable facts. In other words, theoretical terms are meaningful to the extent that their values restrict the values of the observables. This view of meaningfulness does not require that the experimental context always be the same. In the examples mentioned above, a value of 'mass' can restrict the observable value of the position of an electron, or a satellite. The same theoretical concept may restrict the value of different observables in different ways, depending on what the experimental context is. Therefore its meaning may depend on such a context. It can be said to be empirically meaningful if, in any case, once the experimental context is specified, it always imposes a constraint on observable magnitudes.

In order to show the effect of theoretical terms, without being committed to the

8 This is the argument for not considering Bridgman's 'operational definitions' as definitions. See Kamlah (1996). 
existence of theoretical entities, and save the essential dynamic characteristic of a theory, Ramsey suggested a way to exhibit the way it imposes a structure upon observables. This constraint on observables should be expressible only in terms of observables. Ramsey proposed a procedure through which theoretical terms do not appear, and are replaced by what is called their Ramsey sentence, which consists in the replacement of the theoretical terms of a finitely axiomatized theory by bound variables.

If we have a theory $T$ containing terms such as "molecule", "mass", "pressure", etc., we can write this theory:

$$
T\left[t_{1}, t_{2}, \ldots, t_{\mathrm{m}}\right]
$$

Then we can replace each of these terms with variables, bound by existential quantifiers:

$$
\left(\exists x_{1}\right)\left(\exists x_{2}\right) \ldots T\left[x_{1}, x_{2}, \ldots, x_{\mathrm{m}}\right] \quad(2),
$$

which is the Ramsey sentence of $T$. This formulation says that there are classes of entities, which have between them special relationships, and the terms of $T$ have been replaced by general existential sentences. With such a procedure, the terms of the theory are neither eliminated nor explicitly defined, but rather implicitly defined (we define in $T$ the relationships between the $x$ terms).

If we were to "Ramseyfy" all the predicates of a theory (all the terms in (1)) we will only get an information about the cardinality of the domain of the theory, and no structural information, and the Ramseyfication is then trivial. Prior to Ramsey's paper, Newman had already addressed such a criticism against Russell's suggestion (see Russell, 1927) that physics only be interested in structures, in relations between objects whose 
existence needn't be assumed:

Any collection of things can be organised so as to have the structure $W$, provided there are the right number of them. Hence the doctrine that only structure is known involves involves the doctrine that nothing can be known that is not logically deducible from the mere fact of existence, except ("theoretically") the number of constituting objects. (Newman, 1928, p. 144)

If then we produce a Ramsey sentence of all the terms of $T$, the only condition required is the cardinality of the domain. But a constraint on cardinality is not a constraint at all. Indeed, elements of a given domain can be arranged in the ordered tuples so that the domain exhibits the structure $W$, and the claim that there is a certain relation such that it generates this structure on a given domain follows from the mere fact that there are enough individuals in the domain. ${ }^{9}$ To explicate how Russell's intention to describe physical realities only in terms of purely structural relations is trivial, Newman writes that:

if an aggregate $A$ consists of objects of which nothing is known but their existence (...) it cannot, I think, be shown that there is a system of "real" relations with the field $A$ and structure $W$. Even the assigning of names in such an aggregate is difficult to justify. We cannot say "Let this be $a$ and that $b$," for there is no possible of explaining the reference of "this" and that". (Newman, 1928, p. 145)

There Newman's criticism is aimed at Russell's "agnosticism" towards the physical

world. ${ }^{10}$ For a Ramseyfication to be other that trivial, "we may assume, then that at least

one possible structure $W$ is known; and this provides the means of naming the events."

(Newman, 1928, p. 146). In other words, to avoid triviality, the domain should already be structured by definite relations. ${ }^{11}$

9 This is a logical property, according to which every set $A$ determines a structure that contains all subsets of $A$, and then every relation in extension on $A$. Therefore one can never fail to generate the structure considered, and such a relation does not say anything.

10 As expressed by Russel in these terms, "The only legitimate attitude about the physical world seems to be one of complete agnosticism as regards all but its mathematical properties.” (Russell, 1927, p. 270)

11 The same remark has been made more recently by Friedman and Demopoulos (1985), and recalled by Ladyman (1998) so as to discard the relevance of Ramsey's method in the structural realism debate. 
Now, following Campbell, among $t_{1}, t_{2}, \ldots, t_{\mathrm{m}}$, we can distinguish between observational terms, $o_{1}, o_{2}, \ldots, o_{\mathrm{n}}$ (such as "red", "iron", "speed $s "$, for instance: they are individual constants, primitive constants, function constants, etc.) and theoretical terms $h_{1}, h_{2}, \ldots, h_{\mathrm{p}}$ (such as "electron", "mass", for instance). contrary to observational terms, theoretical terms $h_{1}, \ldots, h_{\mathrm{p}}$ do not receive an immediate signification through observation. These theoretical terms have with the observational terms specific relationships, given in $T:$

$$
T\left[o_{1}, o_{2}, \ldots, o_{\mathrm{n}}, h_{1}, h_{2}, \ldots, h_{\mathrm{p}}\right]
$$

The Ramsey reduced form of (3) is:

$$
\left(\exists X_{1}\right)\left(\exists X_{2}\right) \ldots\left(\exists X_{\mathrm{p}}\right) T\left[o_{1}, o_{2}, \ldots, o_{\mathrm{n}}, X_{1}, X_{2}, \ldots, X_{\mathrm{p}}\right]
$$

In (4), theoretical terms have been replaced by general existential sentences where the terms do not denote any more theoretical entities, but specify the relation between these entities and observations. $X_{1}, \ldots, X_{\mathrm{p}}$ variables are higher-order variables and, provided that (3) is a first-order sentence, (4) is a higher-order sentence.

Distinguishing an observational language and a theoretical language is a way to assume such a primal structure that Newman requires. This distinction is not obvious and might be a matter of practical choice, or depend on the theoretical context considered. ${ }^{12}$ "Atom" could be sorted among the theoretical terms but one can arguably say that they can be observed through the use of an electronic microscope. The point here is not to draw a line between observational and theoretical terms, but rather to see whether, if such a distinction is made, it can allow to address the criticism of triviality against Ramsey's 12 See also Maxwell (1962) on the vagueness of the predicate "observable." 
method. How such a distinction can be made, i.e., on what basis one can consider something to be an observable property or fact, has been discussed, among others, by van Fraassen (1980), Ian Hacking (1983), Jody Azzouni (1997 and 2004) or David Lewis (1980). Bueno gives the following set of sufficient (but not necessary) conditions, adapted from the works of Azzouni and Lewis:

An object is taken to exist if we have an access to this object such that: (1) The access is robust (it doesn't depend on our beliefs about the object). (2) It can be refined (we can get closer to the object for a better look). (3) The access allows us to track this object (in space and time). And (4) the access is counterfactually dependent on the object (if the object weren't there, we wouldn't observe it). (Bueno, 2010, p. 98)

There, he put forth conditions of existence as object, but they may be used as conditions of observability. ${ }^{13}$ For instance, an electron does not satisfy this set of conditions, since one cannot "get closer" to it, but certain patterns in a cloud chamber would. These conditions are debatable, but it is not the object of this work. It is assumed that a distinction can be made between unobservable, theoretical entities, and what serves as an empirical, evidential basis. What I wish to discuss is that, provided such a distinction be made, an ontological commitment towards observables does not imply a further commitment towards unobservable entities.

Assuming that such a distinction can be made, we want to show that Ramseyfication can be not trivial, i.e., that by Ramseyfying theoretical terms only, by reducing (3) into (4) we put a constraint on the class of models $M$ for (3)

$$
M\left(o_{1}, o_{2}, \ldots, o_{\mathrm{n}}\right)
$$

13 Thereby, he abandons Quine's identification of quantifier identification of quantifier commitment and ontological commitment. I use these set of conditions as a criterion for what is observable (and not what exists), and then need not abandon Quine's criterion for ontological commitment. 
in terms of observables only (i.e., it shows how introducing theoretical terms gives a constraint to (5) to be embeddable into (3)).

In the Ramseyfied form, no theoretical term appears, and the observational consequences, as well as explanatory and predictive power, are the same as before. It looks like there is a reduction in the sense that theoretical terms have been eliminated from $(3) \cdot{ }^{14}$

To show how the elimination of terms can impose such a constraint, let us consider the following result, presented in Tuomela (1973, pp. 43-44), after Craig (1960, p.103).

Consider a first-order predicate calculus $(\mathcal{L})$, a theory $T$ is here any set of formulas which is closed under deduction in $(\mathcal{L})$, and a basis in $(\mathcal{L})$ for $T$ is any subset $B$ of $T$ such that the closure under deduction of $B$ in $(\mathcal{L})$ is $T$. A proper sub-theory of $T$ is any set $T$ such that for set $\mu=\left\{X_{1}, X_{2}, \ldots, X_{\mathrm{k}}\right\}$ of non-logical predicate symbols (the set of all the theoretical terms for instance), $T$ consists of those formulas in $T$ from which the members of $\mu$ are

14 It is to be noted that not all terms are Ramsey eliminable. Sneed showed it by means of examples: Let $T(\{O\} \cup\{P\})$ be a theory where $P$ is a two-place theoretical predicate, and $O$ a two-place observational predicate. The axioms of $T(\{O\} \cup\{P\})$ are:

(i)

(ii)

(iii)

(iv) $(\forall x)(\forall y)(P x y \rightarrow(\exists z)(O x z) \&(\exists z)(O y z))$

$(\forall \mathrm{x})((\exists z)(O x z) \rightarrow(\exists y)(P x y \&(w)(P x w \rightarrow w=y)))$

$(\forall x)((\exists z)(O x z) \rightarrow(\exists y)(P y x \&(\forall w)(P w x \rightarrow \mathrm{w}=\mathrm{y})))$

$(\forall x)(\forall y)(O x y \& O y z \rightarrow O x z)$.

The sentences of $T(\{P\} \cup\{O\})$ are true only in models $\{D, P, O\}(O, P \subseteq D \times D)$ in which there is one-one correspondence between individuals which stand in the first place in the $O$-relation and those which do not. It can be shown that there is no (finite or infinite) set $T$ of sentences, containing only the predicate $O$ and identity, which is true in exactly those models $\{D, O\}$ where such a correspondence exists. Thus, $P$ is not semantically Ramsey-eliminable in the theory $T(\{O\} \cup\{P\})$. (See Sneed (1971, p. 54) and Tuomela (1973, pp. 60-61)) However, this does not prevent us from seeing how Ramsey elimination can yield the semantical import of terms, and then be a tool for identifying the meaning of theoretical terms by showing the constraint they put on observable terms. 
absent. Then we have the following: let $T$ be any theory, and $C$ any consequence of $T$, the largest subtheory of $T$ in which members of $\mu=\left\{X_{1}, X_{2}, \ldots, X_{\mathrm{k}}\right\}$ do not occur. Then there are a recursive basis $B$ of $T$ and a recursive basis $B^{\prime}$ of $T$, such that $B^{\prime}$ is obtained from $B$ by eliminating the members of $\mu=\left\{X_{1}, X_{2}, \ldots, X_{\mathrm{k}}\right\}$ and $B^{\prime}+C$.

However, this result of Craig's replacement program cannot apply as such to our present discussion. Indeed, theoretical terms are not really eliminated, as long as they still appear through the value of the $X_{1}, \ldots, X_{\mathrm{n}}$ bound variables. In the Ramsey sentence, the theoretical term may not appear anymore. But such a change is only formal since, from a semantic point of view, they still appear as values of the second-order variables. Then not only do we need to show how this result could apply in the case of second-order Ramsey sentences, but also should we worry about the bloating of the ontological commitment of theories. ${ }^{15}$ As such, Ramseyfication does not constitute a satisfactory operation because of its higher-order nature. Going from (3) to (4) introduces second-order quantification. It is not clear what role second-order quantifiers play, and, moreover, since (4) puts in play quantification over second-order predicates, which include theoretical ones, their existence is assumed.

15 This was in fact for Hempel the main reason for which the use of Ramsey sentences would be objectionable:"[Ramsey's method] is perhaps the most satisfactory way of conceiving the logical character of a scientific theory, and it will be objectionable mainly, or perhaps only, to those who, on philosophical grounds, are opposed to the ontological commitments involved in countenancing variables that range over domains other than that of the individuals of the theory (...)." (Hempel, 1965, p. 221) 


\section{First-Order Ramsey Sentences: a Successful Elimination of Theoretical Terms.}

Maintaining the observational / theoretical distinction, we can show that, with adequate rules for a first-order logic, it is possible to give a first-order sentence equivalent to a Ramsey-sentence. This would prove Ramsey's original consideration toward universals, ${ }^{16}$ and give a satisfying justification for a pragmatic conception of theories, which does not need to consider the issue of the nature or reality of theoretical entities, and yet address their usefulness in the process of scientific investigation. ${ }^{17}$ Second-order existential quantification is problematic, because 1) it does not eliminate theoretical terms (which are still present as a value of the second-order variable), 2) it involves a second-order quantification that raises an empiricist worry of the reality of theoretical entities, and potentially in a greater extent that without Ramsey elimination (through the many possible values of second-order variables). However, if we can express second-order quantifiers (the ones used in (2) or (4)) into a first-order logic, then Ramseyfying all theoretical predicates in a theory would avoid assuming their existence, since they could be shown as equivalent to a quantification over first-order entities (observables). Ramseyfication, as we saw, would then show the effect of the import of theoretical terms on the choice of models for the theory, in terms of observables only.

Now, we can see that it has been shown that second-order existential quantifiers can be expressed in first-order logic. We need an extended first-order logic, in which we

16 See his paper "Universals."

17 This can be considered as a justification of Sahlin's assertion that Ramsey "advocates a form of theoretical instrumentalism [in "General Propositions and Causality"]." 
can express relations of dependency and independence. This extended first-order logic, introduced by Hintikka and Sandu (1989), has been also named Independence Friendly logic, or IF logic. To the classical first-order logic, it adds an independence indicator, written '/'. For instance, in the formula $\forall x \forall y(\exists z / \forall y) R(x, y, z), \exists z$ is syntactically subordinate to both $\forall x$ and $\forall y$, but is marked as independent of $\forall y$, and is then dependent only on $\forall x$. Dependency and independence refer here to functional dependency between quantities in a model. For example,

[t]he kinetic energy of a material body depends on its mass and its speed, but does not depend on the particular material body being considered. This fact can be expressed in IF logic by the sentence $\forall b \forall m \forall v(\exists e / \forall b) \mathrm{R}(b, m, v, e)$, where ' $b$ ' ranges over material bodies, ' $m$ ' over real numbers measuring mass, ' $v$ ' over real numbers measuring speed, ' $e$ ' over real numbers measuring energy and ' $R(b, m, v, e)$ ' is read ' $e$ is the kinetic energy of $b$ having mass $m$ and speed $v$.' (Tulenheimo, Stanford Encyclopedia of Philosophy, 2009)

The interest of IF logic is that with it, we can express relations of dependence between variables that the classical first-order logic cannot express.

An important result is that with such an extended logic we can express secondorder existential quantifiers in a first-order logic. ${ }^{18}$ To see how this is true, let us first put a second-order sentence in its prenex normal form, i.e., write it as a string of existential quantifiers followed by a quantifier-free part. A formula in its prenex form is logically equivalent to its original form. In the case of a second-order sentence, its prenex normal form consists of a string of second order quantifiers followed by a first-order formula. Every second-order sentence can be written as a $\Sigma^{1}{ }_{1}$ formula, which consists of a string of second-order quantifiers followed by a $\Sigma_{0}^{0}$ formula (a first-order formula). Let us

18 The following demonstration of this property is adapted from (Hintikka, 2009). 
consider the Ramsey reduced form of a theory, seen earlier:

$$
\left(\exists X_{1}\right)\left(\exists X_{2}\right) \ldots\left(\exists X_{\mathrm{p}}\right) T\left[o_{1}, o_{2}, \ldots, o_{\mathrm{n}}, X_{1}, X_{2}, \ldots, X_{\mathrm{p}}\right] \text {. }
$$

And let us consider a Ramsey reduced form of any sentence of this theory:

$$
\left(\exists X_{1}\right)\left(\exists X_{2}\right) \ldots\left(\exists X_{\mathrm{q}}\right) S\left[o_{1}, o_{2}, \ldots, o_{\mathrm{m}}, X_{1}, X_{2}, \ldots, X_{\mathrm{q}}\right] \text {, }
$$

where $S$ is a quantifier-free first-order formula. This is a $\Sigma^{1}{ }_{1}$ sentence. In it, we can replace all predicates by function variables, and obtain a formula of the form:

$$
\left(\exists f_{1}\right)\left(\exists f_{2}\right) \ldots\left(\exists f_{\mathrm{q}}\right)\left(\forall o_{1}\right)\left(\forall o_{2}\right) \ldots\left(\forall o_{\mathrm{m}}\right) S\left[o_{1}, o_{2}, \ldots, o_{\mathrm{m}}, f_{1}, f_{2}, \ldots, f_{\mathrm{r}}\right] .
$$

The original theoretical terms $\left(t_{1}, \ldots, t_{\mathrm{q}}\right)$ had been replaced by second-order variables $\left(X_{1}\right.$, $\left.\ldots, X_{\mathrm{q}}\right)$, which we have here replaced by function variables $\left(f_{1}, \ldots, f_{\mathrm{q}}\right)$, where $f_{\mathrm{i}}\left(o_{\mathrm{k}}\right)=o_{1}$. Thus, we consider that theoretical terms can be replaced by functional dependencies between observables. Let us assume a simple case in which we have the following formula:

$$
\text { ( } \exists f)(\forall o) G[o, f(o)] \text {. }
$$

Here, it is assumed that $f$ does not appear nested. Now (9) is equivalent with the following formula:

$$
\left(\forall o_{1}\right)\left(\forall o_{2}\right)\left(\exists o_{3} / \forall o_{2}\right)\left(\exists o_{4} / \forall o_{1}\right) \&\left(\left(o_{1}=o_{2}\right) \supset\left(o_{3}=o_{4}\right) \& F^{*}\right)
$$

Here $F^{*}$ is like $F$ except that $A(f(o))$ has been replaced by $(\forall w)\left(w=o_{3} \supset A(w)\right)$ and $f(o)=$ $a$ by $(\forall w)\left(w \supset o_{3} \supset w=a\right)$.

This equivalence can be shown to hold as follows: The Skolem function translation into second-order form is

$$
\left(\exists f_{1}\right)\left(\exists f_{2}\right)\left(\forall o_{1}\right)\left(\forall o_{2}\right)\left(\left(o_{1}=o_{2}\right) \supset\left(f_{1}\left(o_{1}\right)=f_{2}\left(x_{2}\right)\right) \& F^{* *},\right.
$$


where $F^{* *}$ is like $F^{*}$ except that $f_{2}\left(x_{2}\right)$ replaces $o_{3}$. Now the first conjunct in (11) says that $f_{1}$ and $f_{2}$ are the same function. Hence (11) and (10) are equivalent to (9).

This elimination is generalizable to any number of initial and existential quantifiers, and then for the general formula (8).

Thus, we have a first-order equivalent to our initial second-order sentence. In (10) we only have first-order existential quantifiers, with which we can express the Skolem function of second-order variables. This shows that, maintaining the theoretical / observable distinction, we can have a successful Ramsey-elimination, that allows for first-order truth-values. This also shows that theoretical entities, even eliminable in such a way, continue to exist through their Skolem functions that introduce them, expressing functional dependencies between observables.

\section{Consequences of the Success of Ramsey-Elimination.}

The challenges to the Ramsey method that have previously been identified can now be overcome. First, the remarks on how the elimination of certain terms can put a constraint on the remaining terms (Craig's replacement program) can now apply to Ramsey sentences: it can be shown how theoretical terms put a constraint on observable consequences.

Moreover, the possibility of Ramsey-elimination does more than just say that a 
theory can have the same observable consequences without certain theoretical terms. It does so by specifying which relations subsist between these observations: through the Skolem functions that specify functional dependencies between observables, it says which definite structure exists in the domain. Therefore, this characterization of the way by which Ramsey-elimination can be achieved counters Newman's argument against their triviality. Or, rather, it fulfills his demand for a structure to exist in order to have nontrivial Ramsey-elimination. Indeed, Ramsey-eliminations are not trivial since they imply definite relations between observables previously established, and require to put the second-order Ramsey sentence in its normal prenex form (i.e., write it as a string of existential quantifiers followed by a quantifier-free part).

It is then more than the observable / theoretical distinction that is assumed as a structure. The Ramsey sentence allows one to exhibit how a given term imposes a constraint on other terms, through its elimination, thanks to functional dependencies within a given domain. Therefore it can be said that it shows the meaning of theoretical terms, it exhibits their effects in limiting the range of possible observable outcomes of a theory.

Regarding the question of the ontological commitment of theoretical terms, this result does not allow us to say that Ramsey sentences really eliminate theoretical terms. They rather show how, in a theory, they play a role in terms of observable consequences. This method does not allow one to conclude that they can be dispensed with. This was not Ramsey's intention, and was already Hintikka's conclusion in 1998 when he wrote 
that the fact that

concepts are imported into our discourse not only by higher-order quantifiers, but also by dependent first-order quantifiers (...) should put to rest for good the idea that Ramsey sentences offer an elimination of theoretical concepts in any natural sense of the term. (Hintikka, 1998, pp. 302-303)

My intention to see in what way this result could serve an empiricist characterization of the meaning of theoretical sentences was not to argue for the irrelevance of theoretical or unobservable terms. It was rather to show that through the use of such terms, one is not bound to believe in the reality of the entities they posit, and then to show that the possibility to obtain first-order Ramsey sentences allows for a characterization of the meaning of theoretical sentences that is compatible with the empiricist principle to "withhold belief in anything that goes beyond the actual, observable phenomena." (Van Fraassen, 1980, p. 202).

This possible reduction allows one to counter an opposition to the scientific realist conception according to which the reality of theoretical entities is asserted the irreducibility of the truth-conditions of assertions that contain them, or, as we saw earlier:

Semantic realists treat t-discourse as having truth-conditions which are irreducible in that the referred-to entities themselves are irreducible and the truth-conditions of an assertion as a whole are not reducible to evidence - or verification conditions. (Psillos, 1999, p. 14-15)

If we ask the question of the meaningfulness of theoretical sentences, and if we assume that such sentences can only be meaningful relative to a given domain with definite relations between observables, then we see that even though the role of theoretical entities is still present, their meaning can still be reduced to first-order conditions, and it 
is possible to reduce the truth conditions of a theoretical sentence to its truth conditions on observables. Therefore, it is

possible not to quantify over theoretical entities, and thus it is possible not to give them truth-values but rather to remain agnostic as to the existence, or reality, of the theoretical entities. In other words, even though the assumed reality and statement of phenomena may be dependent upon theoretical assumptions, a further ontological commitment is yet not necessary, since no truth needs to be assessed other than the one of the terms referred to as observable.

\section{Conclusion.}

Empiricism may very well require that, for a theoretical term to be meaningful, it should only say something about the observables, that is, impose a constraint on possible empirical results. To that effect, Ramsey's method of formal elimination of theoretical terms can be an interesting tool. It has faced important logical objections: its triviality if no preliminary structure is assumed, and its use of second-order quantification. The latter had mostly been addressed with respect to the problem of the ontological commitment it involved, but the fact that it appealed to second-order logic had generally been overlooked. I have argued that both criticisms can be overcome: solutions have been formulated to show that, provided we use adequate rules of first-order logic, second-order 
Ramsey sentences had a first-order logic equivalent, and that this reduction implies a definite structure, namely functional dependencies between observables (expressed through the Skolem function). Therefore the Ramsey elimination can constitute a relevant way to bring to light the empirical meaning of (sets of) theoretical terms, in way that is consistent with an empiricist attitude toward the reality of unobservable, theoretical entities.

\section{References}

Azzouni, J. (1997). Thick Epistemic Access: Distinguishing the Mathematical from the Empirical, Journal of Philosophy 94, 472-484.

Azzouni, J. (2004). Deflating Existential Consequence. New York: Oxford University Press.

Braithwaite, R. B. (1953). Scientific Explanation, Cambridge University Press.

Bueno, O. (1999). What is Structural Empiricism? Scientific Change in an Empiricist Setting, Erkenntnis $50,59-85$.

Bueno, O. (2010). Structural Empiricism, Again, in Bokulich, A. and Bokulich, P. (eds.), Scientific Structuralism, Boston Studies in the Philosophy of Science, Vol. 281.

Campbell, N. R. (1920). Physics: The Elements, Cambridge University Press (I refer here to the 1957 edition).

Craig, W. (1953). On Axiomatizability within a System. Journal of Symbolic Logic, 18, 30-32.

Craig, W. (1960). Bases for First-Order Theories and Subtheories. Journal of Symbolic Logic, 25, 97-142.

Demopoulos, W. and Friedman, M. (1985) 'Critical Notice: Bertrand Russell's The Analysis of Matter: Its Historical Context and Contemporary Interest', Philosophy of Science 52, 621-639.

Hacking, I. (1983). Representing and Intervening, Cambridge University Press.

Hempel, C. (1965). Aspects of Scientific Explanation and other Essays in the Philosophy of Science. New York: Free Press ("The Theoretician's Dilemma" was first published in 1958 in H. Feigl, M.

Scriven \& G. Maxwell (Eds.), Minnesota Studies in the Philosophy of Science (Vol. 2). Minneapolis: University of Minnesota Press).

Hintikka, J. and Sandu, G. (1989). Informational Independence as a Semantical Phenomenon, in Fenstad, J. E. et al. (eds.), Logic, Methodology and Philosophy of Science VIII, Elsevier Science Pub., 571-5 
89.

Hintikka, J. (1996). The Principles of Mathematics Revisited, Cambridge University Press.

Hintikka, J. (1998). Ramsey sentences and the Meaning of Quantifiers, Philosophy of Science, Vol. 65, 2 (Jun. 1998), 289-305.

Hintikka, J. (2009). A Proof of Nominalism: an Exercise in Successful Reduction in Logic, in Hieke, A. and Leitgeb, H., Reduction - Abstraction - Analysis. Proceedings of the 31th International Ludwig Wittgenstein- $\quad$ Symposium in Kirchberg, 2008, Verlag.

Jammer, M. (2000). Concepts of Mass in Contemporary Physics and Philosophy, Princeton University Press.

Ladyman, J. (1998). What is Structural Realism? in Studies in History and Philosophy of Science 29 (3), 409-424

Laudan, L. (1981). A Confutation of Convergent Realism, in Philosophy of Science, Vol. 48 (1), 19-49.

Lewis, D. (1980). Veridical Hallucination and Prosthetic Vision, in Australasian Journal of Philosophy 58, 239- 249.

Maxwell, G. (1962). The Ontological Status of Theoretical Entities, in Feigl and Maxwell, Scientific Explanation, Space, and Time vol. 3, Minnesota Studies in the Philosophy of Science, 3-15.

McMullin, E. (1984). A Case for Scientific Realism, in Scientific Realism, Jeplin, J. (ed.), U. of California Press .

Newman, M. (1928). Mr Russell's “Causal Theory of Perception”. Mind, Vol. 37, No. 146 (Apr. 1928).

Putnam, H. (1975). Mathematics, Matter and Method, Vol. 1. Cambridge University Press.

Psillos, S. (1999). Scientific Realism, How Science Tracks Truth, Routledge.

Ramsey, F.P. (1929). Theories, repr. in D. H. Mellor (ed.) Foundations: Essays in Philosophy, Logic, Mathematics and Economics, London, Routledge \& Kegan Paul.

Russell, B. (1914). Our Knowledge of the External World, reimpr. 1969, London: GeorgeAllen and Unwin Ltd.

Russell, B. (1927). The Analysis of Matter, New York: Dover Pub. (I refer here to the edition of 1954).

Sahlin, N.E. (1990). The Philosophy of F.P. Ramsey, Cambridge University Press.

Sneed, J.D. (1971). The Logical Structure of Mathematical Physics, Dordrecht: Reidel.

Tulenheimo, T. (2009). Independence Friendly Logic, in The Stanford Encyclopedia of Philosophy, Edward N. Zalta (ed.), URL $=<\underline{\text { http://plato.stanford.edu/entries/logic-if } />}$

Tuomela, R. (1973). Theoretical Concepts, Library of Exact Philosophy 10, Springer-Verlag, Wien and New York.

Van Fraassen, B. (1980). The Scientific Image, Oxford: Clarendon Press.

Worrall, J. (1989). Structural Realism: The Best of Both Worlds?, in Dialectica 43, 99-124. 